First publ. in: Psychological Reports 33 (1973), pp. 471-484

\title{
GROUP-INDUCED EXTREMIZATION: REVIEW OF EVIDENCE AND A MINORITY-CHANGE EXPLANATION ${ }^{1}$
}

\author{
HELMUT LAMM, GISELA TROMMSDORFF, AND EDITH ROST-SCHAUDE \\ Universität Mannbeim, West Germany
}

\begin{abstract}
Summary.-A review of evidence from a number of experiments suggests that as a result of discussion the average of the group members' positions (attitudes, judgments) becomes more extreme. These shifts seem to occur primarily when group members' initial positions are distributed across the two sides of the scale in such a way as to create a majority-minority constellation. Accordingly, it is proposed that underlying the extremity-shifts reported in the literature is the movement of a minority toward the majority's side. That such minority-change is not the only source of extremization is suggested by one study finding that group discussion enhances the extremity of individual positions. An analysis of the special distribution of positions existing on the items selected in that experiment permits the following conclusion. Discussion-induced extremization is (also) caused by the impact of arguments in the discussion favoring the side which most or all members preferred to begin with. Other explanations cannot be discarded at the present time. At the least they point to important aspects of small-group functioning which it would seem fruitful to investigate.
\end{abstract}

There is now evidence from more than a dozen experiments suggesting that a small group context (discussion) leads to an extremization of positions (judgments, attitudes). Thus, "extremity shifts" is a chapter heading in Insko and Schopler's (1972) recent text in experimental social psychology.

The present article provides a review of the relevant evidence, providing an idea of the conditions under which group-induced extremity-shifts have been found to occur. Then, after identifying one particular condition as present in all reports of extremity-shift, a minority-change explanation of the phenomenon is offered. Other existing explanations are briefly noted.

\section{Method Typicaldy Used in Relevant INVESTigations Stimulus Material and Response Task \\ $S$ is asked to indicate his position concerning a statement (e.g., to indicate the extent to which he agrees or disagrees) or a hypothetical person (e.g., to in- dicate the extent to which he evaluates him as favorable or unfavorable). Table 1 shows the various kinds of stimulus materials and response scales used in the studies published up to now.}

\section{Procedure Used to Compare Individual and Group Conditions}

The typical experiment compares initial, individual responses made in the absence of any group interaction, with final, group responses, made unanimously

This paper was written at the Sonderforschungsbereich für Sozial- und Wirtschaftspsychologische Entscheidungsforschung, Universität Mannheim, with financial aid from the Deutsche Forschungsgemeinschaft. 


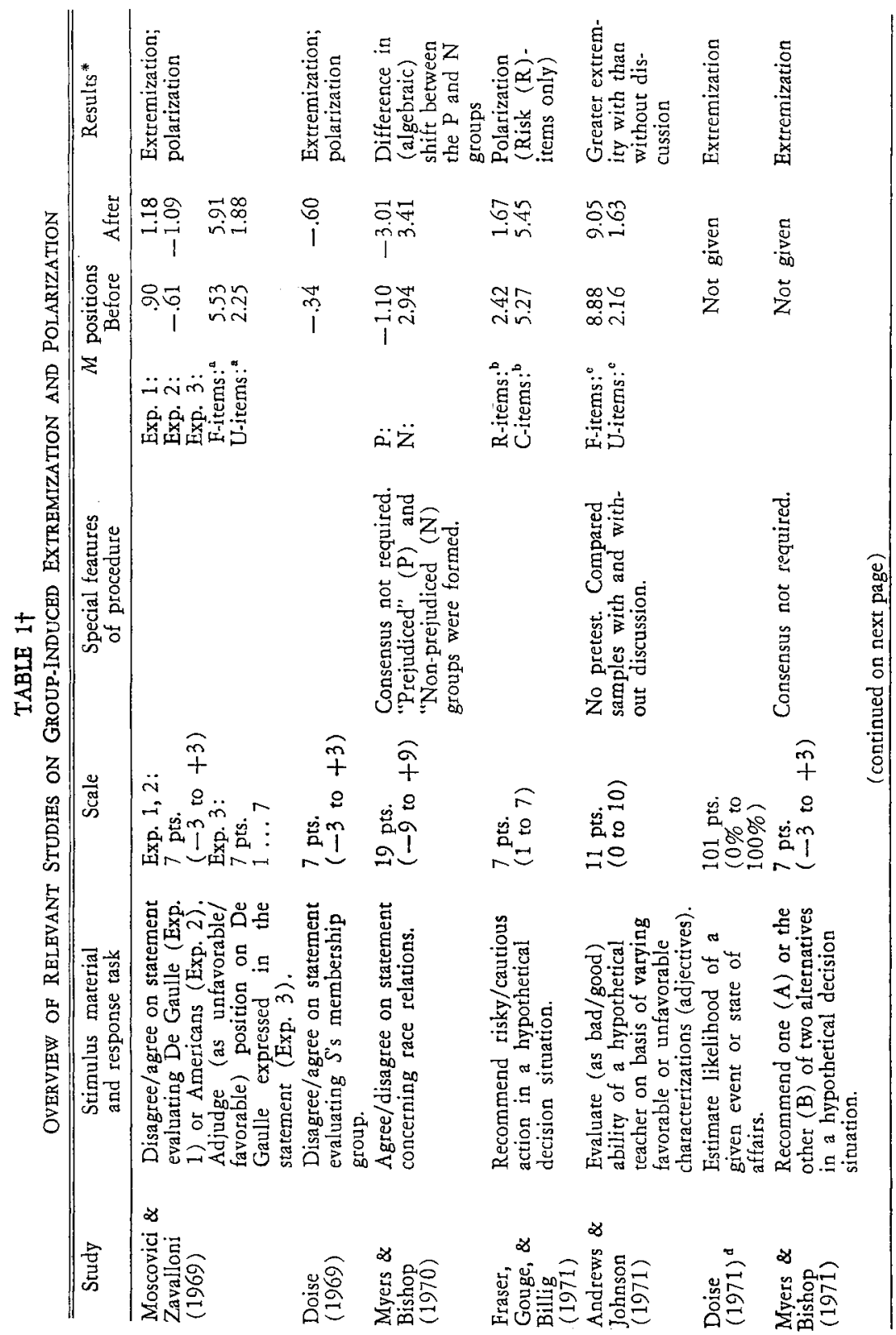




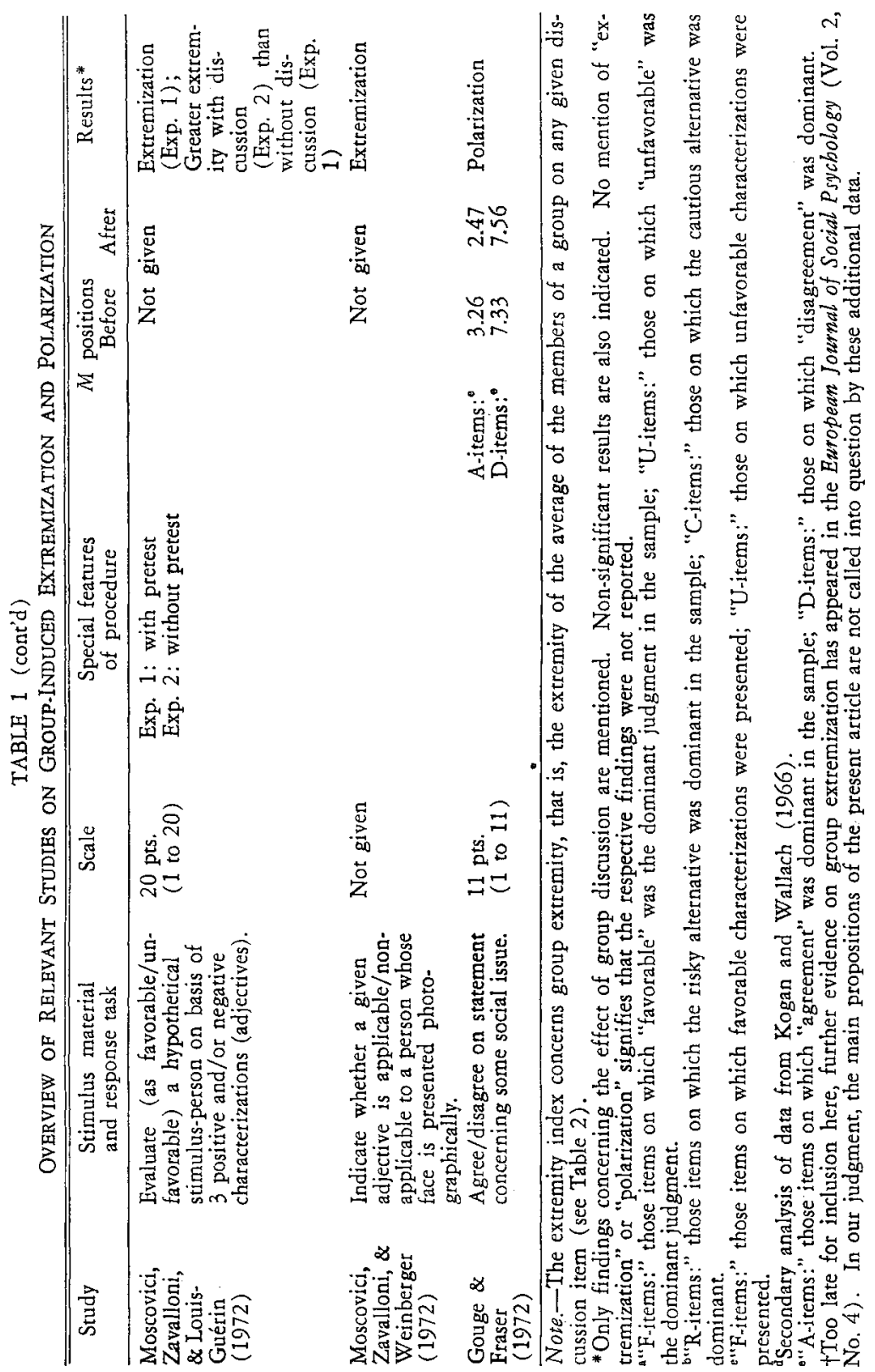


by the group after discussion. In other words, in the typical experiment a small group of $S_{s}$ (usually four) are asked to discuss each item of the stimulus material and reach a consensus on the given question (unanimous position on the scale). Table 1 notes those studies in which this basic paradigm was modified, e.g., no consensus required.

\section{Measures}

Extremity and extremization (see Table 2).-The extremity of a scale position is its distance from the neutral (or mid-) point. In asking whether the group members' post-discussion, relative to their pre-discussion, positions on a given item are more extreme ("extremization") or less extreme ("de-extremization"), the difference between the first and the second measure must be obtained, and these measures must be group scores, i.e., based on the group as the unit of analysis. In calculating pre-discussion extremity, all studies to be reported here used the extremity of the group average (the mean of the group members on a given item), following the example of Moscovici and Zavalloni (1969). Postdiscussion extremity is the extremity of the unanimous group position (or, if no consensus is required, again the extremity of the group average). Thus, extremization in the present paper refers to the increased extremity of real or constructed group positions. (The group-induced extremization of individual positions is considered toward the end of the paper.)

Dominance (or polarizedness) and polarization.- Suppose the task is for each $S$, in a sample of 48, to indicate his agreement or disagreement with a statement on a seven-point scale ranging from 1 (agree strongly) to 7 (disagree strongly). Then the "agree" attitude (or side, or pole) is said to be dominant in the sample if the sample's average is located on the "agree" side-that is, between 1.00 and 3.99-and the "disagree" attitude is dominant if the sample's average is located on the "disagree" side of the scale. Thus, an attitude (or side, or pole) is the more strongly dominant in a collectivity (sample) the nearer its average position is to the respective pole.

"Polarization" refers to an increase-from the first to the second measurement-in the proximity of the sample's average to the dominant pole or, in other words, an increase in the extent to which an attitude is dominant in a sample. (It may be noted that this use of "polarization" in the relevant literature is somewhat specialized, as in general usage the term may also refer to the mere state of being polarized or also to bi-polarization, as in the polarization of a community.)

Distinguishing between "extremization" and "polarization."-Both terms, extremization and polarization, have been explained here because both have been used in the literature and because the two respective indices may yield results of different statistical significance, though empirically they will be highly correlated. Suppose that on one of 10 items in a sample of 48 (12 four-person groups), using a seven-point scale, the sample's pre-discussion average is .82 
TABLE 2

INDICES OF EXTREMIZATION IIIUSTRATED BY EXAMPLE OF A FOUR-PERSON GROUP

\begin{tabular}{cccc}
\hline Response type & Scale* & Extremity of positions \\
\cline { 3 - 4 } & & Group & Individual \\
\hline Pre-discussion responses & & & 5 \\
Member A & +2 & & 2 \\
Member B & +1 & & 1 \\
Member C & -2 & & 2 \\
Member D十 & +1.50 & 1.50 & 2.50 \\
Group score $(M)$ & & & 5 \\
Post-discussion responses & +5 & & 2 \\
Member A & +2 & & 1 \\
Member B & +1 & & 0 \\
Member C & 0 & 2.00 & 2.00 \\
Member D & 2.00 & & 0 \\
Group score $(M)$ & 0 & & 0 \\
Shifts in responses§ & 0 & & 0 \\
Member A & 0 & & +2 \\
Member B & -2 & & 0.50 \\
Member C & -0.50 & -0.50 & +0.50 \\
Member D & & & \\
Group score $(M)$ & & & \\
\hline
\end{tabular}

*This example assumes an 11-point scale ranging from -5 to +5 (algebraic positions) †According to our definition this member constitutes the minority.

\#t is assumed here that only the minority changes its response, in the direction of the majority (thus illustrating the typical movement posited by our "minority-change" explanation).

$\S$ Pre- minus post-discussion responses. Positive values indicate a decrease, negative values an increase in extremity.

and it becomes 1.15 following discussion. This is a polarization effect whose statistical significance is determined by forming, for each of the groups, the algebraic before-after difference and testing whether the average difference score (over the 12 groups) is significantly different from zero. Now suppose that in one group the four members' pre-discussion positions average -.50 and their post-discussion positions average (or their consensus is) -1.00 . This extremization would add to the total extremization index of the sample. Yet it would detract from the total polarization index of the sample, due to the fact that this particular case is somewhat deviant from the rest, going in the opposite of the generally dominant pole. This example shows that a significant extremization will be easier to obtain than a significant polarization, as there may always be some cases in which group members' positions happen to be at variance with the tendency that is dominant in the whole sample on a given item.

In the following, when referring to the shift phenomenon in a general way, we will use the term "extremization," as it ultimately denotes the more "micro" case of a group discussing an item, whereas "polarization" has a characteristic (average position) of the whole sample bound up in it. 


\section{EVIDENCE OF GROUP-INDUCED EXTREMIZATION}

Each of the 10 published studies reports a finding to the effect that positions on an issue become more extreme and/or polarized through group discussion concerning that issue (see Table 1 for details). That this extremization or polarization is not due to any effect of pre-testing has been shown by Andrews and Johnson (1971) and by Moscovici, Zavalloni, and Louis-Guérin (1972). That it is not due merely to the repetition of measures has been shown in those studies that included a control condition, finding that a test-retest without group discussion had no effect (Doise, 1971; Myers \& Bishop, 1970, 1971).

In all studies, with the exception of Myers and Bishop (1970, 1971), the group participants were required to reach a consensus. However, some provided for a third, post-consensus, individual measurement and found that the extremization or polarization effects were maintained even beyond the discussion situation (Moscovici \& Zavalloni, 1969; Doise, 1969; Fraser, Gouge, \& Billig, 1971).

\section{Explaining Extremization EFfect on Basis of Minority-CHANGe}

It is proposed that the group-induced extremization and polarization effects reviewed above (Table 1 ) are attributable in large part to processes whereby a minority changes its position toward the position of the majority of the group. To demonstrate the plausibility of our interpretation we will show, first, that a majority-minority constellation (as defined below) is a frequent case in the relevant experiments; second, why in such a case minority movement toward the majority amounts to an increase in extremity or polarization (given the particular indices explained above); and, third, the central question of why in such a case the minority is likely to change toward the majority.

\section{Definition and Prevalence of Majority-Minority Constellation}

Definition.-A majority-minority constellation is said to be present if the initial positions of group members regarding a given item are distributed such that more of them are located on one side, e.g., agree, than on the other side (disagree) or than on the neutral (mid-) point of the scale. Thus, in the example of Table 2, a majority-minority constellation is given as only one group member's initial position is on the minus-side whereas the three other positions are on the plus-side.

Otber possible constellations.-There are three other possible constellations, i.e., distributions of initial positions in a group on a given item: (a) an equal number of members are on each side (e.g., two are "pro" and two are "con"); (b) all members are on the mid-point; and (c) all members are on one side (e.g., all are "pro").

Prevalence of the majority-minority constellation.-Consider first the kind of study in which there is no significant degree of dominance (initial polarizedness) on any item in the experimental sample. Assume a notmal distribution 
of positions in the sample around the mean (i.e., mid-point of the scale) on each item. ${ }^{2}$ Even under these conditions it is likely that in a fair proportion of discussion cases there will be a majority-minority constellation (if groups are composed at random). Of course, the most frequent case will be that in which an equal number are on both sides (constellation a).

Consider now the kind of study in which a marked degree of dominance is given on some or all of the items in the experimental sample. (In a number of the studies, this was the case either by chance or by preselection of items.) Assume a normal distribution of positions in the sample around the mean on any item. Here, the initial positions in a given group are likely to be distributed in such a way that more of them are located on one side (the one that is dominant in the whole sample) than on the other (or than on the mid-point). Thus, the majority-minority constellation is typically the case under these conditions, and it will be the more frequent on an item the more dominance that item elicits in the sample.

\section{Why Minority Change Amounts to Extremization}

Consider the example in Table 2. Suppose that (as in this example) as a result of group discussion only the minority moves from its initial position (on the minus-side) - in the direction of the majority (plus-side) - whereas those in the majotity do not change. The result is that the group average moves further toward the plus-pole or, in other words, it becomes more extreme. Thus, movement by the minority toward the majority implies group extremization. (An exception is the rare case where the initial group average lies on the side of the minority, which is possible when the minority is very extreme and the majority is very moderate. In this case the group average becomes less extreme as a result of the minority moving toward the majority.)

\section{Why Minority is Likely to Move Toward Majority}

Informational inducement.--It is likely that in the majority-minority constellation here considered more arguments are emitted in a group for the majority's side than for the minority's stand. (Some arguments of the majority will be repetitive in content; but some will be different from each other and be new to one or the other member; and a few arguments may come out that are new to all group members, relative to their individual pre-discussion cogitations on the respective issue.) This informational bias provides one possible inducement for the minority to change toward the majority. In effect it is an instance of attitude change in the direction favored by the source of the communication. (That this informational bias may also lead majority members to change, toward their preferred pole, will be considered later.)

\footnotetext{
${ }^{2}$ This is a conservative assumption in relation to our argument that extremization here is caused by minority change, as compared to the alternative assumption that the distribution of positions is skewed in the sense of higher frequencies toward the preferred pole.
} 
Group pressure as experienced by the minority.-In addition, the minority is likely to conform: it will move toward the majority because it feels that the latter expect that (see Kiesler \& Kiesler, 1969, p. 33). Here the underlying motivational forces may be the desire to be accepted, (the duty) to help fulfill the group's goal (teach a consensus), and/or the intrinsic aversiveness of interpersonal disagreement.

In short, it is suggested that the minority's movement toward the majority is the outcome of one or both of two social-psychological processes (or principles): adjustment of judgments in response to information, and conformity in response to real or imagined group pressure.

\section{Completing the Argument}

We have tried to show that given a majority-minority constellation the minority is likely to move toward the majority, resulting in (group) extremization. We have also shown that majority-minority constellations are likely to be given even in experiments not characterized by dominance and that they are the typical case in experiments characterized by dominance. Thus extremization in the context here described is explained-and to be expected-on the basis of minority change, if it is assumed that the other possible three constellations of initial positions ate not conducive (or susceptible) to extremization or deextremization. In our view the latter assumption can be plausibly made for the first two constellations (ali on mid-point or equal number on each side). As to the third constellation (all on one side), there is reason to expect an extremization (see the final sections of this article) and thus we submit that minoritychange is just one-but not the only-source of extremization.

\section{Explanattons Proposed in Published Studies}

In this section we briefly note the various explanations offered by investigators in interpreting their findings. Each type of explanation will be noted only in connection with the author(s) who first proposed it. Supporting evidence will be mentioned only if based on a statistical test.

Moscovici and Zavalloni (1969) believe that their extremization and polarization findings are caused by the presumed enhancement, through group discussion, of (a) participants' involvement with the issues under consideration, (b) participants' certainty about their positions, and/or (c) the meaningfulness of the task.

Doise (1971) considers "cognitive restructuring" as underlying extremization: "Individuals . . a arrive at differentiated judgments, whereas groups are categoric" (Doise, 1969, p. 143). In line with this proposition is the finding that fewer response categories (points of the rating scale) were used in postdiscussion than in pre-discussion judgments (Moscovici \& Zavalloni, 1969). However, it is possible that this finding was due simply to the consensus requirement and is a reflection of the convergence of positions. 
Fraser, Gouge, and Billig (1971) note various possible mechanisms by which polarization may come about. One of them has to do with the distribution of initial positions in a group; in particular, a skewed distribution is said to favor polarization. In a similar direction goes the suggestion by Andrews and Johnson (1971) that the greater polarization observed in their groups may be a "climb-on-the-bandwagon" effect caused by group members discovering that most of them are leaning in the same direction on a particular question" (p. 192). (Our minority-change explanation goes in the same direction but specifies the processes through which the distribution of initial positions in a group may eventuate in extremization.)

As Fraser, Gouge, and Billig (1971) note, another possible explanation of polarization is that variations in the initial positions of group members may be associated with variations in such attributes as involvement and certainty (or confidence). (Stroebe and Fraser (1971) have confirmed earlier evidence that extremer positions are indeed correlated with greater confidence.) One piece of relevant evidence is Doise's (1969) finding, from postexperimental questionnaire data, that the "extremist" was perceived by the group to have been more influential and more certain about his positions than would have been predicted by chance. ${ }^{3}$ However, these correlations may have been due to the possibility that group members, at the end of the discussion sessions, were aware of their shift toward greater extremity and hence attributed greater influence to the person who had yielded least in the group discussions.

Myers and Bishop (1971) propose what they call the "mutual-reinforcement" explanation of extremization, which posits a preponderance of discussion arguments in favor of the dominant side. In support of this interpretation they report that in their experiment an average of $76 \%$ of the arguments were in favor of the side which was dominant in the sample and also a positive correlation between the percentage of "dominant" arguments and polarization. The process ultimately underlying the extremity shift, according to this interpretation, is the reinforcement of a pre-existing tendency toward a favored (dominant) pole through the reward provided by the statement of an attitude similar to one's own.

While our minority-change interpretation also implies a preponderance of arguments (and/or stated positions) for the majority in a particular case of group discussion, it does not invoke the relatively complex similarity- (attractiveness-) rewardingness-reinforcement chain but posits simply adjustment to information and/or conformity. ${ }^{4}$

\footnotetext{
"These findings are significant only in the "experimental condition" where accompanying $S_{\mathrm{s}}$ ' task of rating their own membership group was the task of estimating the opinions of an outgroup concerning $S_{s}$ ' membership group.

"Myers and Bishop (1971) do not specify why the reinforcement of one's attitude should imply its extremization, except by equating attitude with "response tendency" toward a pole of the scale whose strengthening leads to greater approachment of the pole ("goal").
} 


\section{EXPLAINING REPORTED RELATIONSHIPS INVOLVING EXTREMIZATION}

Only three studies have reported statistically significant (or near-significant) relationships involving extremization as a (dependent) variable.

Doise (1969) found that extremization (of opinions concerning one's membership group) was stronger ( $p<.06$, one-tailed) in a condition where an outgroup (students of a rival school) was symbolically present (i.e., $S$ s had to estimate their rival group's opinions of them). This finding can be accommodated by our minority-change interpretation in one or both of two ways. First, as Doise's (1969) data show, initial positions (opinions) were already more extreme and more polarized (though probably not significantly so) in the outgroup condition. This means that probably there were more marked minority-majority constellations and thus stronger conditions for minority change in the outgroup condition. In addition, increased pressure toward uniformity (quick consensus) may, as Doise (1969) suggests, have been due directly to the "solidarizing" effect of a salient outgroup. (This is suggested by the fact that the difference between the normal and the outgroup conditions was not upheld in post-consensus individual responses.) Plausibly, it is the minority (deviants) on which such pressure toward uniformity acts most sttongly.

Doise (1971) reports that extremization was stronger for males than for females but he offers no explanation. In the absence of any data on initial extremity we can only speculate that initial extremity may have been higher for the male groups and hence more marked majority-minority constellations existed in the male sample.

Myers and Bishop (1971) report a positive correlation between initial group average and proportion of dominant arguments and between proportion of dominant arguments and polarization. The correlations were calculated across all $84(=6$ items $\times 14$ groups $)$ discussion cases. This result is in agreement with the "adjustment-to-information" process of our minority-change interpretation. The more marked the majority-minority constellation, the more will the majority dominate the discussion with arguments in favor of their side, and the greater will be the informational inducement acting on the minority to adjust its position.

\section{Discussion-induced Extremization of Individual Positions}

As has been emphasized at the outset, the findings reviewed and explained above concern the extremization of group positions [that is, the basic (group) index involves the extremity of the average position (or consensus) of the group members, concerning a given item].

However, it is also of interest to consider the influence of group discussion on the extremity of individual positions. Here, the basic (group) index involves the average of the extremity scores of the group members concerning a given item (see Table 2). Using this index of individual extremity, Kogan and 
Wallach (1966) found no extremization. On the contrary, on the basis of (nonsignificant) results they conclude that "the ovet-all findings clearly point to an extremity decline as a consequence of group discussion to consensus" ( $p$. 168).$^{5}$ In contrast, Myers and Bishop (1971) found an increase in the extremity of individual positions through group discussion (without consensus). Our task now is to explain the latter finding.

It is important to note that Myers and Bishop (1971) used only items involving dominant attitudes (that is, given a scale from -3 to +3 , only items on which the sample averaged below -1 or above +1 were retained for group discussion). This means that there were few discussion cases in which all group members were located on the neutral point or an equal number were on both sides. ${ }^{6,7}$ In other words, the principal question is, why did an extremization of individual positions occur in the (presumably typical) constellation in which the initial positions of most or all of the group members were on the same side?

Considering first the former case-the majority-minotity constellation-it may be noted that our explanation of group extremization via minority change is applicable to individual extremization only if the minority's initial position is located on the neutral point. Otherwise, movement by the minority from its side (X) toward the majority side (Y)-assuming no other change of positions in the group-results in a decrease of individual extremity (except in the unlikely yet interesting case in which the former minority comes to take a more extreme position on side $Y$ than it did formerly on side $X$ ).

A change by the minority away from the majority toward an even more extreme position would result in an increase in average individual extremity, assuming no other change of positions in the group. This is probably a rather untypical outcome, though such possibilities of "reactance" or "anticonformity" are not altogether to be discarded. It may be noted, too, that the experimental instructions of Myers and Bishop (1971), with no consensus requirement, would permit such anti-majority movement by the minority, whereas the consensus requirement in Kogan and Wallach's (1966) experiment precluded that possibility.

It can be concluded that the extremization of individual positions in the type of case under consideration, i.e, most or all initial positions are on one side, is

\footnotetext{
These data were the same on which Doise (1971) after a secondary analysis found an increase in the extremity of group positions (see Table 1). The picture is similar in the case of McCauley (1970), who found an extremization of group positions but no change in the extremity of individual positions.

${ }^{\circ}$ In contrast, Kogan and Wallach (1966) and McCauley (1970) - who found no extremization of individual positions-did not use only items on which dominant judgments or attitudes existed.

${ }^{7}$ In these two cases we assume that no shift occurs. However, it is conceivable that in the second case an over-all individual extremization occurs by virtue of a movement of members on each side closer toward their preferred pole. Such a "bi-polarization" could be expected especially when the two subgroups are easily identified, e.g., by pre-existing attributes distinguishing them from each other.
} 
produced by the majority (pro-Y) members becoming more extreme on their side. What are the underlying social-psychological processes?

One process - which has much in common with the first process mentioned in our minority-change explanation of group extremization-is the adjustment to new information. Starting from the assumption that a preponderance of discussion arguments favor the majority side (Y) - for which Myers and Bishop (1971) provide evidence-it is suggested that some of the arguments produced are new to some of the members, allowing the latter to move toward a more unreserved (extreme) position on their favored side of the issue.

A pro-Y member who voices pro- $Y$ (majority) positions and/or arguments will be reinforced in that activity. In other words, the verbal and/or nonverbal rewards he receives from other pro-Y members (e.g., in the form of approval) will lead him to repeat the rewarded behavior. As a result, he will voice new arguments or enlarge on previous ones. Reinforcement in this sense will provide one reason as to why an "information bias," the basis for the informational inducement underlying extremization, will be present in the group discussion.

In addition, reinforcement may underlie extremization in a more direct way, i.e., not through the information link. If group member $A$ is rewarded (through approval) by other pro-Y members for a moderate pro-Y statement, then he may expect even stronger or surer reward to follow a stronger (more extreme) pro-Y statement. Assuming a need for (the appearance of) consistency between one's (verbal) behavior and one's attitude, the result will be an extremization of one's position.

Two other possible interpretations, which have been mentioned previously with respect to group extremization, are also applicable to individual extremization: increased involvement through group discussion, and a disproportionately strong influence of extreme participants in the discussion.

\section{CONCluding Discussion}

In order to obtain some indication of the generality of discussion-induced extremization it is important to find out empitically in which constellation of initial positions it does or does not occur. Is it empirically true that all or most group members must be on one side of an issue for an extremization to occur? Only a comparative analysis of extremization in the various constellations in an experiment can provide an answer.

How does (a) the majority-minority constellation compare with (b) the constellation in which all group members are on one side? Is group extremization really stronger in the former than in the latter case? If so, this points to the viability of our minority-change explanation since none of the other explanations could account for the difference.

Even more precise investigation is possible by studying the movement of individual group members. If in case (a) it is only (or primarily) the minority 
that shifts toward the majority pole, then this is proof that minority-change is indeed responsible for the increase in group-extremity. However, the underlying factors need to be determined. In particular, it remains to be shown whether the experience of group pressure plays a significant role in the minority's movement toward the majority. Conceivably, that is only the case given a consensus requirement. (Everyday unorganized discussion groups usually are not under a consensus requirement.)

If there is a shift by (majority) members toward the pole they initially prefer it is proof that minority change is not the (only) source of discussion-induced extremization. The task then remains to seek an empirical answer to the following questions concerning the role of various possible causes of extremization (of majority members). (a) Do pro- $Y$ arguments, i.e., those for the majority side, outnumber pro- $X$ arguments, and are new arguments generated through discussion? [Affirmative evidence on the first part of the question exists (Myers \& Bishop, 1971).] If so, is the extremization of group members caused merely by the impact of information or (also) by positive reinforcement coming from other group members? (b) Does participants' involvement in the issues really increase through group discussion? If so, by which intervening processes does the increase in involvement lead to an increase in extremity? (c) Do more extreme group members participate more actively (with more contributions, with greater intensity) in group discussion? Are they actually more "expert" at the issues (put out a greater variety of supporting facts and argue more cogently for their positions) or is such expertness only ascribed to them by other group members, e.g., because extremer members may appear more certain or talk more? Devising appropriate testing procedures will be the task for experimental investigators interested in these questions.

Why would it be worth investing in empirical research of the sort just outlined to identify the sources of discussion-induced extremization? For one thing, our understanding of small-group processes will be enriched by more knowledge on the comparative influence of moderate versus extreme members, the effect of group discussion on participants' involvement with the respective issue, and the mutual reinforcement processes taking place in group discussion. These topics have been investigated largely in the context of attitude change, with a passive recipient and an active (but "programmed") source of communication. Research on these topics in the small-group context will be a step toward greater relevance to the real world and generalizability of the knowledge obtained.

Uncovering the processes underlying the extremization effect, thus identifying the sufficient and necessary conditions for its occurrence, will help in making informed hypotheses about the occurrence of the phenomenon in real-life group contexts.

On the basis of the laboratory findings reviewed here, all that can now be stated is that the discussion-induced extremization of attitudes or judgments oc- 
curs only in situations where most of the group members are on the same side of an issue to begin with. (Thus, to speak generally of an "extremity shift" would be misleading.) And our analysis suggests that the extremization found consists largely in an assimilation of initially deviant members to the majority of the group. Myers and Bishop's (1971) finding of a discussion-induced extremization of individual positions-for which a replication would be desirable-is important because it suggests that, even if the group members are initially agreed on the side of the issue that is "right," some of them come to endorse that side more extremely through group discussion. If upheld in further research, this is a rather new discovery of an empirical principle which should be of interest to social-science analysts and to those who observe and participate in small groups.

\section{REFERENCES}

ANDREwS, I. R., \& JOHNSON, D. L. Small group polarization of judgments. Psychonomic Science, 1971, 24, 191-192.

DOISE, W. Intergroup relations and polarization of individual and collective judgments. Journal of Personality and Social Psychology, 1969, 12, 136-143.

DOISE, W. An apparent exception to the extremization of collective judgments. European Journal of Social Psycbology, 1971, 4, 511-518.

FrAser, C., GoUgE, C., \& BILLIG, M. Risky shifts, cautious shifts and group polarization. European Journal of Social Psychology, 1971, 1, 7-30.

GOUGE, C., \& FRASER, C. A further demonstration of group polarization. European Journal of Social Psycbology, 1972, 2, 95-97.

INSKO, C. A., \& SCHOPLER, J. Experimental social psychology: text with illustrative readings. New York: Academic Press, 1972.

KIESler, C. A., \& Kiesler, S. B. Conformity. Reading, Mass.: Addison-Wesley, 1969.

KOGAN, N., \& WALlACH, M. A. Modification of judgmental style through group interaction. Journal of Personality and Social Psychology, 1966, 4, 165-174.

MCCAUley, C. R., JR. Risk and attitude shifts after group discussion. Unpublished doctoral dissertation, Univer. of Pennsylvania, 1970.

Moscovicr, S., \& ZAVAlionI, M. The group as a polarizer of attitudes. Journal of Personality and Social Psychology, 1969, 12, 125-135.

Moscovici, S., ZaValloni, M., \& LouIS-GuÉRIN, C. Studies on polarization of judgments: I. Group effects on person perception. European Journal of Social Psycbology, 1972, 2, 87-91.

Moscovici, S., Zavalloni, M., \& WeINBerger, M. Studies on polarization of judg. ments: II. Person perception, ego involvement and group interaction. European Journal of Social Psychology, 1972, 2, 92-94.

MYERS, D. G., \& BISHOP, G. D. Discussion effects on racial attitudes. Science, 1970, 169, 718-719.

MYERS, D. G., \& BISHOP, G. D. Enhancement of dominant attitudes in group discussion. Journal of Personality and Social Psychology, 1971, 20, 386-391.

STROEBE, W., \& Fraser, C. Riskiness and confidence in Choice Dilemma decisions. European Journal of Social Psycbology, 1971, 1, 519-526. 